\title{
A Performance Comparison of CDMA Ad-Hoc and Cellular Networks
}

\author{
Jeffrey Q. Bao \\ Semiconductor Product Sector \\ Motorola Inc. \\ Mansfield, MA 02048 \\ ljb057@email.mot.com
}

\author{
Lang Tong \\ School of Electrical Engineering \\ Cornell University \\ Ithaca, NY 14850 \\ ltong@ee.cornell.edu
}

\begin{abstract}
A performance comparison is presented between two types of CDMA random access systems: cellular and ad hoc. Based on a finite population model, the network throughput is derived for both systems. Two aspects of the performance comparison are addressed: (1) the throughput comparison; (2) the impact of spreading gain and error control coding on the throughput in both systems. Evaluations of these performance comparisons are also provided.
\end{abstract}

\section{INTRODUCTION}

As two distinct types of networks, ad hoc and cellular systems have their own advantages and disadvantages. For narrow-band modulated slotted Aloha wireless networks with fully connected nodes, a successful packet transmission happens only when one packet appears in a time slot, hence classical performance results (for example, results in [4]) can be applied to both cellular and ad hoc types of networks. In CDMA systems, however, multiple packets with different spreading codes can be correctly received simultaneously by different receivers in a time slot (i.e. the multiple access capability of CDMA systems ). Therefore the architecture of a network plays an important role in the network performance.

Many performance studies about CDMA systems have been focused on the physical layer without considering the network architecture and the traffic characteristics [6]. At the link layer, the work by Raychaudhuri [8] about the performance of cellular type of slotted Aloha CDMA networks has been widely applied to different applications [3, 7]. In addition, the impact of capture effect on the network performance of slotted Aloha CDMA cellular systems is investigated in [2]. Polydoros and Silvester proposed a general framework [5] to incorporate the multiple access capability and the capture effect into the analysis of slotted Aloha CDMA systems with dedicated transmitting and receiving nodes.

For ad hoc CDMA systems where nodes transmit directly to each other and any node can be a potential transmitter or receiver, few performance study results are available in the literature. One reason, as suggested in [9], is that the analysis becomes intractable due to the uncoordinated behavior of nodes in such networks. Therefore, the effect of the ad hoc architecture on the network performance has not been investigated. Furthermore, the effects of spreading gain and error control coding on the network performance are not fully understood.

The contribution of this paper is the following:(1) the performance of one-hop slotted Aloha ad hoc systems employing the transmitter-based CDMA scheme is analyzed; (2) the effect of the network architecture on the performance of slotted Aloha CDMA networks is studied by comparing the performance of an ad hoc system with that of a cellular system; (3) impacts of the spreading gain and error control coding on the network performance of both systems, which also lead to the understanding of efficiency of bandwidth utilization in both systems, are investigated.

\section{TWO SYSTEM MODELS}

We consider a local area where a number of nodes are fully connected by a common radio channel. Time is 
slotted, and we assume that each packet requires one time slot to transmit. Two types of network architecture are studied: cellular and ad hoc.

\subsection{The Cellular System}

The first system is a cellular system as shown in the left part of Figure 1. Multiple nodes transmit packets to each other through a Base Station (BS), i.e. nodes transmit packets to the BS via the up-link and the BS broadcasts these packets to potential receiving nodes through the down-link. We assume a Time Division Duplex (TDD) system with equal-sized up-link and down-link periods, each of which is one time slot. Nodes are half-duplex and are always in the receiving mode during the down-link period. During the up-link period, nodes are in the transmitting mode. A slotted Aloha random access protocol is used by all nodes in the up-link: whenever a node has a new packet to transmit, it sends the packet in the earliest available up-link time slot. If the packet is not successfully received by the BS, the node will retransmit the packet with a fixed probability in each successive up-link slots until a successful transmission occurs.

Each node in the network transmits packets in the up-link using an unique spreading code which is assumed to be randomly generated. The BS has the knowledge of each node's code. We assume that the receiver at the $\mathrm{BS}$ is a bank of matched filters. Furthermore, we assume that in the down-link the BS uses different orthogonal codes for packets intended for different nodes so that the transmission success of a packet depends on the up-link reception alone.


Figure 1: Left: A Cellular System; Right: An ad hoc system

\subsection{The ad hoc System}

The right part of Figure 1 illustrates an ad hoc network. Nodes transmit to each other directly through a common channel by which all nodes are fully con- nected. Each node can be a potential transmitter or receiver. A similar slotted Aloha random access protocol as that in the cellular system is also employed by all nodes with the difference that there is no up- and down- link periods. The transceiver at each node is also half-duplex.

As in the cellular system, every node uses a unique code to spread its transmitted packets. In order to receive packets from any potential nodes, we assume that each node has the knowledge of all possible spreading codes and the receiver at each node is also a bank of matched filters.

\subsection{Analysis Assumptions}

Assuming the number of nodes in the network is finite, we follow the convention used in the analysis of slotted Aloha systems by Kleinrock and Lam [4]: a node which needs to retransmit a packet is referred to as in the backlogged state; otherwise a node is in the unbacklogged state. To simplify analysis, we ignore noises and assume that errors in a packet are caused by Multiple Access Interference (MAI) alone. A Linear block code is used for error correction. We make the following four assumptions about both the cellular and the ad hoc system:A1 nodes generate packets according to independent Poisson processes with equal arrival rate $\frac{\lambda}{M}$, where $M$ is the total number of nodes; A2 there is an immediate feedback about the status of the transmission; A3 there is no buffer at any node, i.e. each node can at most hold one packet at a time; A4 with probability $s_{k i}$, the receiver at the BS or a node in the ad hoc system detects successfully $i$ out of $k$ colliding packets in a time slot. For the ad hoc system, we have following additional assumptions: A5 each node has equal probability to transmit to every other node; A6 the transceiver at each node functions independently from other nodes.

\section{THROUGHPUT COMPARISON}

We follow the Markov Chain approach with the number of backlogged nodes $n$ being the network state. The network throughput is defined as the average number of packets successfully received by their intended receivers in a time slot. We focus on key steps in determining the network throughput for both cellular and ad hoc systems, details can be found in [1].

To summarize the multi-packet reception capability 
of CDMA receivers, we define the reception matrix $S$ for each node in the ad hoc system or the BS in the cellular system

$$
S=\left(\begin{array}{ccccc}
s_{10} & s_{11} & 0 & \cdots & 0 \\
s_{20} & s_{21} & s_{22} & \cdots & 0 \\
\vdots & & & & \vdots \\
s_{M 0} & s_{M 1} & s_{M 2} & \cdots & s_{M M}
\end{array}\right)
$$

In the cellular system, every up-link packet is intended for the BS; however, in the ad hoc system, a node can successfully detected a packet not intended for it. For example, as shown in the right part of Figure 1, node $\mathrm{A}$ and $\mathrm{C}$ are transmitting to node $\mathrm{B}$, node $\mathrm{E}$ is transmitting to node D. Due to the full connectivity of the network, three packets are presented in the receiver front end of both B and D. Even D can successfully demodulate one packet, there is only $\frac{1}{3}$ chance that $\mathrm{D}$ gets E's packet. Therefore, $S$ does not characterize the multi-packet reception capability of a node in the ad hoc system. We define a network reception matrix $R$ for the whole network

$$
R=\left(\begin{array}{ccccc}
r_{10} & r_{11} & 0 & \cdots & 0 \\
r_{20} & r_{21} & r_{22} & \cdots & 0 \\
\vdots & & & & \vdots \\
r_{M 0} & r_{M 1} & r_{M 2} & \cdots & r_{M M}
\end{array}\right)
$$

where $r_{j k}$ is the probability that $k$ out of $j$ packets in the time slot are received by their intended receivers in the network. The conversion from $S$ to $R$ in the ad hoc system is given by the following theorem

Theorem 1 Under A1-A6, given total $L \leq M$ packets are transmitted in a time slot, the probability that there are $n \leq L$ successfully received packets by their intended receivers in the network is given by

$$
\begin{aligned}
r_{L n}= & \sum_{l=n}^{L} \sum_{J=m i n(l, 1)}^{\min (l, M-L)} q_{L l} \frac{\left(\begin{array}{c}
M-L \\
J
\end{array}\right)}{(M-L)^{l}} \times \\
& \sum_{\sum_{j=1}^{J} a_{j}=l} \frac{l !}{a_{1} ! a_{2} ! \cdots a_{J} !} \times \\
& \left(\sum_{\sum_{j=1}^{J} b_{j}=n} \prod_{i=1}^{J} d_{L, a_{i}, b_{j}}\right)
\end{aligned}
$$

where $a_{j}=1,2 \cdots l, b_{j}=0,1, \cdots a_{j}$ and

$$
q_{L l}=\left(\begin{array}{c}
L \\
l
\end{array}\right)\left(\frac{M-L}{M-1}\right)^{l}\left(\frac{L-1}{M-1}\right)^{L-l}
$$

$$
d_{L, a_{i}, b_{i}}=\sum_{k=b_{i}}^{L-\left(a_{i}-b_{i}\right)} \frac{\left(\begin{array}{c}
a_{i} \\
b_{i}
\end{array}\right)\left(\begin{array}{c}
L-a_{i} \\
k-b_{i}
\end{array}\right)}{\left(\begin{array}{c}
L \\
k
\end{array}\right)} s_{L k} .
$$

A proof can be found in [1]. In general, $R$ is a function of $S$ and the network traffic pattern.

To determine elements of $S$, we apply the Gaussian assumption about MAI and assume bit errors happen independently. Let $k$ be the total number of packets in a slot, $N$ be the spreading gain, $L_{p}$ be the packet length in bits, the BER $x$ of a packet is given by $x=Q\left(\sqrt{\frac{3 N}{k-1}}\right)$ with $Q(y)=\frac{1}{\sqrt{2 \pi}} \int_{y}^{\infty} e^{-\frac{t^{2}}{2}} d t$. The packet success probability is then $p_{c}(k)=\sum_{i=0}^{t}\left(\begin{array}{c}L \\ i\end{array}\right) x^{i}(1-x)^{L_{p}-i}$ where $t$ is the number of bit errors that can be corrected by coding. If each matched filter works independently at the BS's receiver, $s_{n k}$ is the probability is

$$
s_{k n}=\left(\begin{array}{c}
k \\
n
\end{array}\right) p_{c}(k)^{n}\left(1-p_{c}(k)\right)^{k-n} .
$$

\subsection{Throughput of the Cellular Network}

Given network state $n$, the number of packets successfully received by their intended receivers in two time slots is $N u m=\sum_{k=1}^{M} p_{k}^{c} \sum_{l=0}^{k} l s_{k l}$ where $p_{k}^{c}$ is the probability that total $k$ packets are transmitted in the up-link time slot. Because the throughput $\beta_{\text {cell }}(n)$ and the average throughput $\bar{\beta}_{\text {cell }}$ is defined per time slot, we can obtain them by the following

$$
\begin{aligned}
\beta_{\text {cell }}(n) & =\frac{N u m}{2} \\
\bar{\beta}_{\text {cell }} & =E\left(\beta_{\text {cell }}(n)\right)=\sum_{n=0}^{M} \beta_{\text {cell }}(n) q_{n}^{c}
\end{aligned}
$$

where $q_{n}^{c}$ is the stationary distribution of the network state Markov Chain.

\subsection{Throughput of the ad hoc Network}

Similarly we can have the throughput $\beta_{a d}(n)$ and the average throughput $\bar{\beta}_{a d}$ of the ad hoc system

$$
\begin{aligned}
\beta_{a d}(n) & =\sum_{k=1}^{M} p_{k}^{a} \sum_{l=0}^{k} l r_{k l} \\
\bar{\beta}_{a d} & =E\left(\beta_{a d}(n)\right)=\sum_{n=0}^{M} \beta_{a d}(n) q_{n}^{a}
\end{aligned}
$$

where $p_{k}^{a}$ is the probability that total $k$ packets are transmitted in one time slot in the ad hoc network. 


\subsubsection{A Throughput Bound of the Ad Hoc Network}

When we have the perfect receiver, i.e. all collided packets can be received successfully by a receiver, intuition suggests that the throughput should reach the maximum. Indeed, for cellular systems, when $s_{j k}=$ $\left\{\begin{array}{ll}1 & j=k \\ 0 & j \neq k\end{array}\right.$ for $j=1,2, \cdots M$, every transmitted packets will be received correctly by the BS, therefore the throughput is equal to the arrival traffic. However, for the ad hoc system, the architecture and the half-duplex mode of transceivers impose limits on the throughput. The following theorem provides the performance bounds of an ad hoc system. The proof can be also be found in [1].

Theorem 2 Under A1-A6,

$$
\begin{aligned}
\beta_{a d}(n) \leq & \sum_{k=1}^{M} p_{k} \sum_{l=0}^{k} l q_{k l}=\sum_{k=1}^{M} p_{k} \sum_{l=0}^{k} l\left(\begin{array}{c}
k \\
l
\end{array}\right) \times \\
& \left(\frac{M-L}{M-1}\right)^{l}\left(\frac{L-1}{M-1}\right)^{k-l} .
\end{aligned}
$$

The equality holds iff $S=S_{*}$ where $s_{j k}= \begin{cases}1 & j=k \\ 0 & j \neq k\end{cases}$ for $j=1,2, \cdots M$.

\subsection{Normalization of the Throughput}

Given spreading gain $N$, and coding rate $r_{c}$, the normalized throughput $\beta_{u}$ is given by $\beta_{u}=\frac{r_{c} \beta}{N}$. To determine coding rate $r_{c}$, we apply the Gilbert-Varsharmov lower bound for block codes :given packet length $L_{p}$ and number of correctable errors $t$, we can have the maximum coding rate by the following two steps: (1) $\alpha=\frac{2 t+1}{L_{p}} ;(2) r_{c}=1+\alpha \log _{2}(\alpha)+(1-\alpha) \log _{2}(1-\alpha)$.

\section{THROUGHPUT COMPARISON EVALUATION}

In the following throughput comparisons, we assumed $M=10$ nodes in both the cellular and the ad hoc system, each packet is 1000 bits long (i.e. $L_{p}=1000$ ).

\subsection{Comparison Between the Cellular and the Ad Hoc System}

We evaluated throughput of both the cellular and the ad hoc systems with varying $N$ at a particular $t$. Figure 2 to Figure 4 shows both the actual throughput vs offered load and normalized throughput vs normalized offered load (left: actual; right: normalized; solid line: ad hoc networks; dotted line: Cellular networks) for these two systems with varying error correction capability $(t=0,5,10$ respectively).
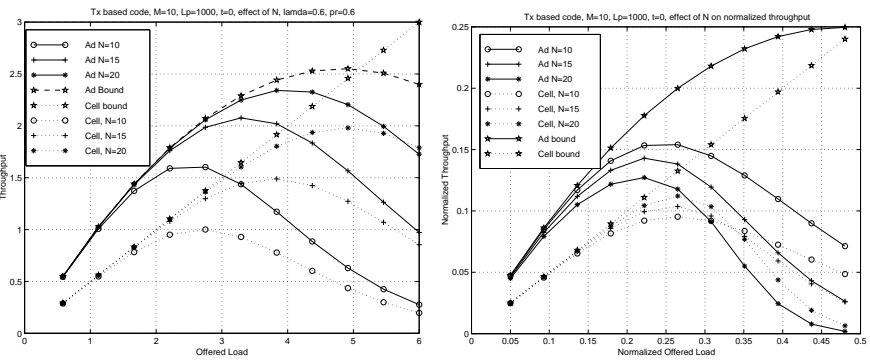

Figure 2: Throughput vs Offered Load for $t=0$ $\lambda=0.6$.
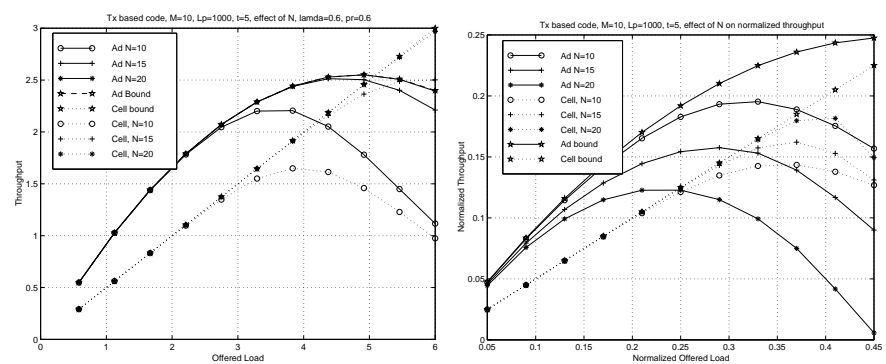

Figure 3: Throughput vs Offered Load for $t=5$ $\lambda=0.6$.
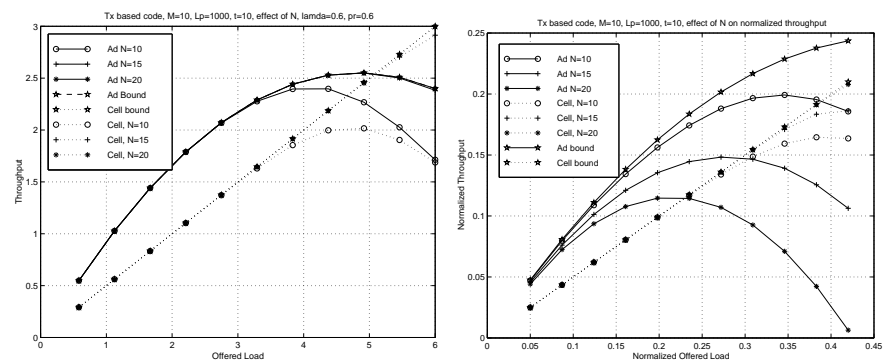

Figure 4: Throughput vs Offered Load for $t=10$

$$
\lambda=0.6 \text {. }
$$

We observed from Figure 3 and 4 that with a moderate powerful receiver ( spreading gain $N>10$ and number of correctable bit errors $t>0$ ), the ad hoc system had higher throughput than the cellular system under light traffic conditions (offered load $<4.5$ ); but under heavy traffic conditions ( offered load $>4.5$ ), the cellular system out-performed the ad hoc system. In cellular systems with a poor performance receiver ( see Figure 2 with $t=0$ ), the advantage of no throughput bound can not show up because under heavy traffic 
conditions, most of the packets can not be received by the BS; hence the ad hoc system still out-performed the cellular system.

\subsection{Effects of Spreading Gain on the Through- put}

We observed from Figure 2 to 4 that in cellular systems, larger $N$ lead to higher normalized throughput except when $t=0$ and normalized throughput was greater than 0.35 . For ad hoc systems, in contrast, larger $N$ actually lead to smaller normalized throughput. Apparently in the cellular system, the throughput improvement was large enough to offset the bandwidth expansion introduced by the increased spreading gain before the normalized offered load reached certain threshold after which the bandwidth expansion became the dominant factor in determining the normalized throughput ( see Figure 2). In the ad hoc system, the bandwidth expansion can not overcome the limitation on the throughput imposed by the node availability, and the normalized throughput actually decreased with increasing $N$.

\subsection{Effects of Error Control on the Through- put}

Next we compare the throughput of these two systems with a fixed $N$ at different $t$ and investigate effects of error control on the throughput for both systems. Figure 5 shows both the actual and the normalized throughput vs offered load for these two systems with $t=0,5,10,15$ at $N=10$. For the cellular system, because the bandwidth expansion caused by larger $t$ is smaller than that caused by increasing $N$, both the normalized and the actual throughput increased as $t$ became larger. Although it is not surprising that more powerful error control will improve the actual throughput in the ad hoc network as shown in the left part of Figure 5, the relationship between $t$ and normalized throughput turns out to be interesting. As can be observed from the right part of Figure 5, the normalized throughput increased for all normalized offered load when $t$ was increased from 0 to 5 . But as $t$ was increased from 5 to 15 , larger $t$ did not necessarily lead to higher normalized throughput.
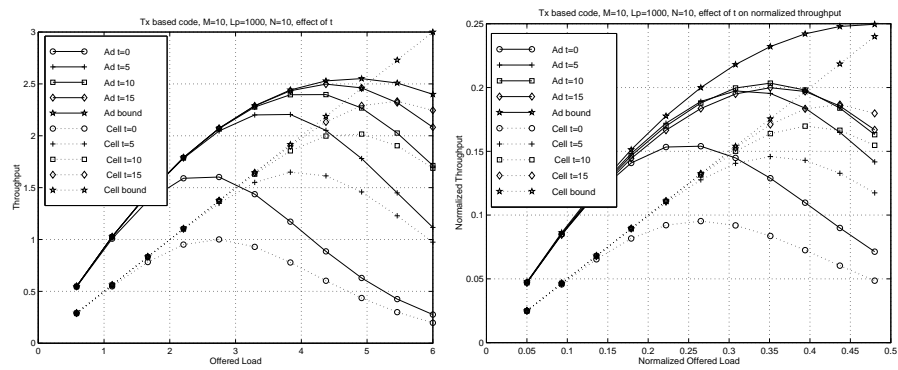

Figure 5: Throughput vs offered load, $N=10$.

\section{REFERENCES}

[1] J. Q. Bao and L. Tong. "Performance Analysis of Slotted Aloha Random Access Ad-Hoc Networks with Multipacket Reception". Submitted to IEEE Trans. Commun., 1999.

[2] D. H. Davis and S. A. Gronemeyer. "Performance of Slotted Aloha Random Access with dealy capture and randomlized time of arrival". IEEE Trans. Comm., COM-28, 1980.

[3] R. K. Morrow Jr. and J. S. Lehnert. "Packet Throughput in Slotted Aloha DS/SSMA Radio Systems with Random Signature Seuences". IEEE Trans. Commun., 40(7):985-1002, July 1992.

[4] L. Kleinrock and S. S. Lam. "Packet Switching in a Multiaccess Broadcase Channel: Performance Evaluation". IEEE Trans. Comm., COM-23(4):410-423, April 1975.

[5] A. Polydors and J. Sylvester. "Slotted Random Access Spread-Spectrum Networks: An Analytical Framework". IEEE Journal on Selected Areas in Communications, SAC-5(6):989-1002, July 1987.

[6] M. B. Pursley and D. J. Taipale. "Error Probabilities for Spread-Spectrum Packet Radio with Convolutional Codes and Viterbi Decoding". IEEE Trans. Commun., COM-35(1):1-12, Jan. 1987.

[7] P. Rapajic. "Performance Analysis of Slotted Aloha/CDMA System with Adaptive MMSE Receivers". IEICE Trans. Fundamentals, E80-A(12):356362, Dec. 1997.

[8] D. Raychaudhuri. "Performance Analysis of Random Access Pakcet-Switched Code Division Multiple Access Systems". IEEE Trans. Comm., COM-29(6):895-901, June 1981.

[9] E. Sousa and J. Silvester. "Spreading Code Protocols for Distributed Spread-Spectrum Packet Radio Networks". IEE Trans. Commun., 36(3):21-29, March 1988. 